\title{
Consensus report of the task force on standardisation of DNA flow cytometry in clinical pathology
}

\author{
M.G. Ormerod ${ }^{\mathrm{a}, *}$, B. Tribukait ${ }^{\mathrm{b}}$ and Walter Giaretti ${ }^{\mathrm{c}, * *}$ \\ a 34 Wray Park Road, Reigate, RH2 ODE, UK \\ ${ }^{\mathrm{b}}$ Department of Medical Radiobiology, Karolinska Institute, Box 60212, S-104 01 Stockholm, Sweden \\ ${ }^{\mathrm{c}}$ Laboratory of Biophysics, Istituto Nazionale per la Ricera sul Cancro, Viale Bendetto XV, $n .10$, \\ 16132 Genova, Italy
}

Received 29 July 1998

Revised 30 July 1998

Accepted 30 July 1998

Abstract. Guidelines are given to assist the standardisation of DNA flow cytometry in clinical pathology. They have been agreed by a group of twelve scientists from nine European countries.

Keywords: DNA, flow cytometry, standardisation, ploidy, S-phase fraction

\section{Introduction}

These guidelines were produced at the request of the council of the European Society for Cellular Analytical Pathology. Previously, guidelines have been published by a North American group [1] who also produced consensus reviews of the clinical utility of DNA cytometry in bladder cancer [2], carcinoma of the breast [3], colorectal cancer [4], neoplastic haematopathology [5] and prostate cancer [6].

Normal resting human cells have 46 chromosomes corresponding to about 7 pg DNA per cell nucleus. During proliferation, the DNA content doubles. Cells that are replicating DNA (in S-phase of the cell cycle) will have an intermediate content of DNA. In malignancy, structural and/or chromosomal aberrations are common. Only when the net chromosome number is changed, can deviations in the DNA content from normal be observed, giving rise to DNA aneuploidy. Thus lack of abnormality in the DNA

\footnotetext{
*Corresponding author: Michael Ormerod. E-mail: Michael_Ormerod@compuserve.com.

** On behalf of the DNA Flow Cytometry Task Force of the European Society for Analytical Cellular Pathology. Members of the group: A. Böcking, Institut für Cytopatholgie, Medizinische Einrichtungen, Heinrich Heine Universität, Düsseldorf, Germany; I. Christensen, Finsen Laboratory, Rigshospitalet, Copenhagen, Denmark; J.-L. D'Hautcourt, Clinique St. Joseph, Mons, Belgium; J. Dufer, Laboratoire de Physiologie Cellulaire, Université de Reims, Reims, France; W. Giaretti, Laboratory of Biophysics, Istituto Nazionale per la Ricera sul Cancro, Genova, Italy; J. Lawry, Institute of Cancer Studies, Medical School, Sheffield, UK; A. Orfao, Servicio General de Citometria, Laboratorio de Hematologia, Hospital Clinico Universitario, Salamanca, Spain; M.G. Ormerod, 34 Wray Park Road, Reigate, UK; A. Sampedro, Servicio de Proceso de Imagenes, Universidad de Oviedo, Oviedo, Spain; F. Sansonetty, IPATIMUP, Porto, Portugal; B. Tribukait, Karolinska Institute, Stockholm, Sweden; G.K. Valet, Max-Planck-Institut für Biochemie, Cell Biochemistry Laboratory, Martinsried, Germany.
} 
content does not exclude malignancy or the presence of chromosome abnormalities. It should also be noted that polyploidisation of normal cells during ageing or after tumour therapy results in an increase in DNA content. Loss of DNA by apoptosis or necrosis must also be taken into consideration in the interpretation of DNA histograms.

Measurement of the DNA content of a large number of cells by flow cytometry gives the DNA histogram from which can be derived the cell ploidy and the components of the cell cycle, including the S-phase fraction. Both ploidy and S-phase fraction may have prognostic significance in certain tumours.

\section{Nomenclature for nuclear DNA measurements [7]}

The nuclear DNA content can be measured on slides in selected nuclei by image cytometry (ICM) or non-selectively in a suspension of cells or nuclei by flow cytometry (FCM). The method used must be defined in the report.

In flow cytometry the following terms are frequently used:

Coefficient of variation (CV) which is measured on a peak in the DNA histogram and is given by $100 \times$ standard deviation/mean channel number.

DNA index (DI) which is the mean channel number of the G1 peak of the tumour/mean channel number of the G1 peak of normal cells. A DNA index of 1 corresponds to diploidy (2c).

Ploidy was originally used to refer to the chromosome number. In cytometry, it is used to describe the overall DNA content. Diploid cells have a DNA content of normal cells although their chromosomes may be abnormal. Cells with a DNA index between 1.90 and 2.10 are classified as DNA tetraploid. Peaks outside the tetraploid and diploid range are referred to as DNA aneuploid. The DNA index should be corrected to allow for any variation in the linearity of the amplifier (see Section 6.2 below).

\section{Sample collection and preparation}

Cells from blood, bone marrow, body fluids, irrigations, surgical biopsies, core biopsies and fine needle aspirates can be used. Fresh material, specimens stored by freezing or by fixation or material fixed and embedded for routine histological examination may be used. The best results are usually obtained by using either fresh or frozen material. The advantage to using histopathological material is that a region of interest can be identified by inspection of a conventionally stained section and then selected. A disadvantage is that antibody staining to identify the tumour cells, as opposed to normal stromal cells, cannot be used.

The quality of the sample will depend on how it is collected and handled after collection. When collecting fluid specimens (blood, aspirates, etc.), coagulation may be a problem and the use of an anticoagulant (heparin or EDTA) should be considered. When freezing single cell suspensions or small biopsies the quality of the thawed sample may be improved if a protectant, such as DMSO, is added.

Whenever possible, the tumour cells should be identified by use of a specific antibody.

A sample of the material should be checked by conventional staining and light microscopy to ensure that it contains an adequate number of tumour cells; $20 \%$ has been suggested as the minimum acceptable proportion if a measurement of S-phase is to be made [1]. 


\subsection{Fixation}

Material may be fixed in ethanol or in buffered formaldehyde. Other fixatives often used in routine histopathology, such as Bouins, Zenkers, mercuric chloride, should be avoided. If fixed samples are to be transported to the flow cytometry laboratory care should be taken to fill the sample tube with fixative so that the entire specimen remains immersed, even when the sample is shaken.

If an antibody stain is used to identify the tumour cells (see below), a fixative which preserves the antigen used must be selected. For many cell surface antigens, fixation can only be performed after antibody labelling.

\subsection{Preparation}

Several authors have given protocols for cell preparation for recording DNA histograms from cells [8-14].

The aim is to obtain single cells or nuclei free of clumps with a minimum of debris. The method of preparation will depend on the way in which the sample has been stored. Several different methods have been developed.

1. Fresh or fresh frozen material. For leukaemias or aspirates of, for example, peritoneal fluid, a singlecell suspension can be readily obtained. Solid tumours can be disassociated mechanically in a buffer containing detergent (such as $0.1 \%$ nonidet-P40) which releases the nuclei into suspension [15]. Sometimes trypsin is included to assist tissue disaggregation [16]. Vindeløv et al. have published details of a buffer which stabilises the nuclei with spermidine [16]. Preparation of nuclei precludes the use of an antibody to a surface or cytoplasmic antigen to distinguish tumour from normal cells.

2. Freshly fixed material. (a) Samples fixed in ethanol. (b) Formalin-fixed material. The sample is cut into small pieces $\left(1-2 \mathrm{~mm}^{2}\right)$ and incubated either with pepsin in $0.1 \mathrm{M} \mathrm{HCl}$ or with trypsin to release the nuclei.

3. Material fixed in formalin and paraffin embedded. The methods used are all variations on that originally published by Hedley et al. [17]. A section is cut from a block of paraffin embedded tissue; it should be at least $50 \mu \mathrm{m}$ thick, a thinner section will contain too many sliced nuclei thereby increasing the debris in the DNA histogram. After the section has been dewaxed and brought through ethanol to water, it is treated with a proteolytic enzyme (either pepsin or protease) to release the nuclei. It is advantageous to place the section in a small cassette while carrying out these procedures; this precaution avoids centrifugation, which can clump the nuclei.

\subsection{Stain}

The choice of stain is governed by the flow cytometer in use. If the machine is equipped with a source UV light (mercury arc lamp or UV laser) and measurement is restricted to DNA, 4'6-diamidino-2phenylindole (DAPI) is the stain of choice. This dye, whose fluorescence is enhanced 200 fold on binding to DNA, is DNA specific. If the flow cytometer is only equipped with an argon-ion laser producing light at $488 \mathrm{~nm}$, propidium iodide (PI) should be used. This dye also binds to double stranded RNA and the sample should be treated with RNase before analysis. DAPI-stained samples can be analysed immediately after adding the dye. PI stained samples improve if they are stored at $4{ }^{\circ} \mathrm{C}$ for a few hours (Ormerod, unpublished data).

It is important that there is sufficient stain to guarantee stoichiometry. For propidium iodide, at least $20 \mu \mathrm{g}$ of PI per million cells is recommended. For DAPI, the dye concentration should be at least $5 \mu \mathrm{M}$. 


\subsection{Cell concentration}

The final concentration of cells or nuclei should be about $10^{6} \mathrm{ml}^{-1}$. At a lower concentration, the flow rate of the sample through the cytometer has to be increased which can degrade the CV; if the concentration is higher, there may be insufficient dye to stain the DNA stoichiometrically.

\subsection{Quality of sample preparation}

It is useful if the final preparation is checked under a microscope (preferably a fluorescent microscope). The following points should be checked:

- particle concentration;

- clumping or excess debris;

- that the majority of the nuclei have the appearance of tumour nuclei (for example, are not from granulocytes);

- absence of cytoplasmic remnants attached to nuclei.

\section{Reference standard for DNA}

The ploidy of a sample is calculated by reference to the peak of diploid cells. In a clinical sample (with the exception of some lymphomas and leukaemias, see below) there is nearly always some normal, diploid nuclei present but it can be difficult to identify which peak is from diploid cells. Identification is helped if a standard reference cell has been added to the sample (trout or chicken erythrocytes or both [16]). The reference cells must be processed identically with the sample and must be added to the sample at the earliest possible stage. Reference cells cannot be used with paraffin-embedded material in which it may possible to separate normal and malignant nuclei using light scatter [18]. Identification of the tumour G1 peak can also be made by using a monoclonal antibody to identify specific cell types (see below).

\section{Measurement of DNA}

(1) A linear amplifier should always be used when measuring DNA. The linearity of the amplifier should be checked (for example, using standard fluorescent beads, clumped, fixed lymphocytes, polyploid liver cells, chicken and trout erythrocytes).

(2) The alignment of the instrument should be checked daily by measuring the CV of standard beads or fixed, stained lymphocytes. The CV obtainable will depend on the machine but should be $\leqslant 2 \%$.

(3) The number of channels in the histogram should be at least 512. The PMT voltage should be adjusted so that the G1 peak of normal, diploid cells does not fall below a channel equal to one fifth of the maximum channel number (that is, 200 in a 1024 channel histogram, 100 in 512 channels).

(4) The electronics should be triggered on the DNA fluorescence.

(5) All signals should be collected, including those from debris, above a channel number equal to 1/10 diploid G1 peak channel.

(6) The number of events collected should be sufficient to give 10-20,000 nuclei in the DNA histogram (excluding debris). The more complex the DNA histogram, the greater the number events which should be recorded. If S-phase is being recorded, then there should be at least 100 cells in S-phase region.

(7) In order to obtain the best CV, the flow rate should be kept low (typically 100-300 events/sec). 


\section{Judgement of quality}

\subsection{Coefficient of variation $(C V)$}

The lower the CV of the peaks in the DNA histogram, the better is the quality and the greater is the amount of information that can be derived. A better CV is obtained from fresh than from paraffinembedded material. From fresh material, CVs in the order of $3 \%$ or less should routinely obtained. The quality of paraffin-embedded material depends on the treatment of the specimen in the histopathology laboratory. CVs of less than 5\% should routinely obtained. An estimation of the S-phase fraction should not be attempted if the CV of the G1 peak is $>8 \%$ [1].

\subsection{Linearity}

If the $\mathrm{G} 1$ to G2/M ratio does not fall between 1.95 and 2.05, the linearity of the amplifier should be checked and, if necessary, the appropriate adjustment made (often it will be found that the offset on the amplifier is misadjusted).

\subsection{Debris}

High debris can interfere with the measurement of the S-phase fraction and could also obscure a small hypodiploid peak. If high debris routinely causes a problem, the sample collection and preparation should be re-evaluated. In a small number of cases, an excessive number of necrotic or apoptotic cells can give excessive debris. An estimation of S-phase fraction should not be attempted if the histogram contains $>30 \%$ debris.

\subsection{Clumping}

Excessive clumps in the sample is an indication of poor sample preparation. They should not be a problem in preparations of nuclei. Clumps will be revealed in the DNA histogram by a peak at a channel number three times the channel of the diploid G1 peak. Clumps will also be revealed, and gated out, by pulse shape analysis (see below).

\section{Multiparameter analysis}

\subsection{Pulse shape analysis of the DNA signal}

If a laser is used with beam shaping optics, an analysis of the shape of the signal from the DNA fluorescence is possible and should be performed (for example, see [10]). A cytogram of the peak or width of the DNA signal against area will reveal clumps and abnormally shaped nuclei.

\subsection{Light scatter}

When possible, a cytogram of right angle versus forward light scatter should be displayed. Debris can usually be identified and gated out of the analysis. In a well-prepared sample, the cells from the inflammatory cells in the tumour can often be identified [18]. 


\subsection{Antibody stain}

An antibody labelled with fluorescein can often be used to distinguish normal from tumour cells. With epithelial tumours, the epithelial cells can be identified with a stain for cytokeratin [19-22]. In non-lymphoid malignancies, normal inflammatory cells can be labelled using an antibody against CD45 (leucocyte common antigen) [23]. In lymphoid malignancies, an antibody to an appropriate cell surface antigen needs to be selected, depending on the classification of the tumour [24].

\section{Evaluation and interpretation of the histogram}

The histogram is used to estimate the DNA ploidy of the tumour and its cell cycle parameters (particularly S-phase fraction). If there is appreciable debris or clumping in the sample and it is not possible to improve the histogram by multiparametric analysis (see above), a computer program can be used to correct for these artefacts [25]. Some computer programs estimate the percentage of aggregates and debris lying between the leftmost G1 and the rightmost G2 in the DNA histogram (termed background aggregates and debris, BAD). It is recommended that an estimate of the S-phase fraction should not be attempted if $\mathrm{BAD}>20 \%$ [1]

\subsection{Measurement of ploidy}

(1) A single peak in the DNA histogram corresponding to the channel of normal human cells is defined as DNA diploid. A peak from G2/M cells should also be present in the histogram.

(2) In lymphomas and leukaemias, in which a sample may contain few normal cells, a single peak deviating more than $\pm 5 \%$ from the expected position for diploid cells should be suspected as aneuploid. In the presence of added DNA standard cells or selection of the tumour cells by an antibody (see above), an interpretation of aneuploidy may be confirmed. In their absence, aneuploidy should be reported with an added caution.

(3) If a peak is observed at a position corresponding to $4 \mathrm{c}$ together with a peak at $8 \mathrm{c}$ and S-phase cells between these two positions, the tumour should be reported as tetraploid. In the absence of an additional (peak at $8 \mathrm{c}$ plus S-phase), if the percentage of cells at $4 \mathrm{c}$ exceeds $3 \times \mathrm{SD}$ of the percentage of cells in G2/M in normal tissues, then tetraploidy should be reported with a cautionary note. Precautions should be taken to ensure that the excess of cells in G2/M is not an artefact due to cell clumping (see above).

A tetraploid peak should have a DI within the range 1.90-2.10. A peak falling outside this range should be reported as DNA aneuploid.

(5) Accuracy of determining whether a tumour is DNA diploid will depend on the CV of the G1 peak. The smaller the CV, the smaller is the deviation from diploid that can be detected. Separation of normal from tumour cells will improve the measurement of small deviations from diploid.

\subsection{Evaluation of cell cycle parameters}

A variety of algorithms have been developed to obtain the percentage of cells in G1, S and G2/M phases of the cell cycle from the DNA histogram [25-27]. The reliability of the information will depend on the quality of the DNA histogram. In particular, a low CV, absence of clumping and low debris are important. 
In a histogram with only diploid cells or one clear-cut aneuploid peak, the rectangle model of Baisch is adequate and can be performed by setting regions and using a pocket calculator [10]. Commercial software exists which will attempt an estimate of S-phase fraction in the presence of overlapping polyploid peaks. The reliability of such procedures is unproven.

Different computer packages and different algorithms may give slightly different values of S-phase on the same DNA histogram. To obtain consistent results, the same computer package and the same algorithm should always be used.

\section{Reporting results}

The following minimum information should be reported:

(1) Ploidy, additionally with the DI of all the populations.

(2) CV of the main G1 peak.

(3) S-phase fraction. If the tumour is diploid, a statement should be made as to whether S-phase was measured on the whole sample (including the normal cells) or on the tumour cells alone (identified, for example, by a specific antibody).

(4) Brief comment if necessary (for example, inadequate number of cells, high debris, CV too high, $\% \mathrm{BAD})$.

\section{References}

[1] T.V. Shankey, P.S. Rabinovitch, B. Bagwell, K.D. Bauer, R.E. Duque, D.W. Hedley, B.H. Mayall and L.L. Wheeless, Guidelines for implementation of clinical DNA cytometry, Cytometry 14 (1993), 472-477.

[2] L.L. Wheeless, R.A. Badalament, R.W. deVere White, Y. Fradet and B. Tribukait, Consensus review of the clinical utility of DNA cytometry in bladder cancer, Cytometry 14 (1993), 478-481.

[3] D.W. Hedley, G.M. Clark, C.J. Cornelisse, D. Killander, T. Kute and D. Merkel, Consensus review of the clinical utility of DNA cytometry in carcinoma of the breast, Cytometry 14 (1993), 482-485.

[4] K.D. Bauer, C.B. Bagwell, W. Giaretti, M. Melamed, R.J. Zarbo, T.E. Witzig and P.S. Rabinovitch, Consensus review of the clinical utility of DNA cytometry in colorectal cancer, Cytometry 14 (1993), 486-491.

[5] R.E. Duque, M. Andreef, R.C. Braylan, L.W. Diamond and S.C. Peiper, Consensus review of the clinical utility of DNA cytometry in neoplastic hematopathology, Cytometry 14 (1993), 492-496.

[6] T.V. Shankey, O.-P. Kallioniemi, J.M. Koslowski, M.L. Lieber, B.H. Mayall, G. Miller and G.J. Smith, Consensus review of the clinical utility of DNA cytometry in prostate cancer, Cytometry 14 (1993), 497-500.

[7] W. Hiddemann, J. Schumann, M. Andreeff, B. Barthologie, C.J. Herman, R.C. Leif, B.H. Mayall, R.F. Murphy and A.A. Sandberg, Convention on nomenclature for DNA cytometry, Cytometry 5 (1984), 445-443.

[8] H.A. Crissman and G.T. Hirons, Staining of DNA in live and fixed cells, in: Methods in Cell Biology, Z. Darnzynkiewicz, J.P. Robinson and H.A. Crissman, eds, Vol. 41, Academic Press, San Diego, 1994, pp. 195-209.

[9] D.W. Hedley, DNA analysis from paraffin-embedded blocks, in: Methods in Cell Biology, Z. Darnzynkiewicz, J.P. Robinson and H.A. Crissman, eds, Vol. 41, Academic Press, San Diego, 1994, pp. 231-240.

[10] M.G. Ormerod, Analysis of DNA - general methods, in: Flow Cytometry: a Practical Approach, M.G. Ormerod, ed., 2nd edn, IRL at Oxford University Press, Oxford, 1994, pp. 119-135.

[11] F.J. Otto, High-resolution analysis of nuclear DNA employing the fluorochrome DAPI, in: Methods in Cell Biology, Z. Darnzynkiewicz, J.P. Robinson and H.A. Crissman, eds, Vol. 41, Academic Press, San Diego, 1994, pp. 211-217.

[12] P.S. Rabinovitch, Practical considerations for DNA content and cell cycle analysis, in: Clinical Flow Cytometry: Principles and Application, K.D. Bauer, R.E. Duque and T.V. Shankey, eds, Williams and Wilkins, Baltimore, 1993, pp. 117-142.

[13] K. Steck and A. El-Naggar, Solid tumor DNA ploidy analysis, in: Flow Cytometry Protocols, Methods in Molecular Biology, M.J. Jaroszeski and R. Heller, eds, Vol. 91, Humana Press, Totowa, NJ, 1998, pp. 181-195.

[14] L.L. Vindeløv and I.J. Christensen, Detergent and proteolytic enzyme-based techniques for nuclear isolation and DNA content analysis, in: Methods in Cell Biology, Z. Darnzynkiewicz, J.P. Robinson and H.A. Crissman, eds, Vol. 41, Academic Press, San Diego, 1994, pp. 219-229. 
[15] S.E. Petersen, Flow cytometry of human colorectal tumors: nuclear isolation by detergent technique, Cytometry 6 (1985), 452-460.

[16] L.L. Vindeløv, I.J. Christensen and N.I. Nissen, A detergent-trypsin method for the preparation of nuclei for flow cytometric DNA analysis, Cytometry 3 (1983), 323-327.

[17] D.W. Hedley, M.L. Friedlander, I.W. Taylor, C.A. Rugg and C.A. Musgrove, Method for analysis of cellular DNA content of paraffin-embedded pathological material using flow cytometry, J. Histochem. Cytochem. 31 (1983), 1333-1335.

[18] M.G. Ormerod, J.T. Titley and P.R. Imrie, The use of light scatter when recording a DNA histogram from paraffinembedded tissue, Cytometry 21 (1995), 294-299.

[19] J.T. Bijman, D.J.T. Wagener, J.M.C. Wessels, P. Van den Broek and F.C.S. Ramaekers, Cell size, DNA, and cytokeratin analysis of human head and neck tumors by flow cytometry, Cytometry 7 (1986), 76-81.

[20] P.S. Oud, J.B.J. Henderik, H.L.M. Beck, J.A.M. Veldhuizen, G.P. Voojis, C.J. Herman and F.C.S. Ramaekers, Flow cytometric analysis and sorting of human endometrial cells after immunocytochemical labelling for cytokeratin using a monoclonal antibody, Cytometry 6 (1985), 159-164

[21] J.C. Van der Linden, C.J. Herman, J.G.C. Boenders, M.M. Van de Sandt and J. Lindeman, Flow cytometric DNA content of fresh tumor specimens using keratin-antibody as second stain for two-parameter analysis, Cytometry 13 (1992), 163-168.

[22] S. Wingren, O. Stål and B. Nordenskjöld, Flow cytometric analysis of S-phase fraction in breast carcinoma using gating on cells containing cytokeratin, Brit. J. Cancer 69 (1994), 546-549.

[23] R.J. Zarbo, R.D. Brown, M.D. Linden, F.X. Torres, R.E. Nakhleh and D.S. Schultz, Rapid (one-shot) staining method for two-color multiparametric DNA flow cytometric analysis of carcinomas using staining for cytokeratin and leucocyte common antigen, Am. J. Clin. Path. 101 (1994), 638-642.

[24] A. Orfao, R. Garcia-Sanz, M.C. López-Berges, M.B. Vidiales, M. González, M.D. Cabellero and J.F. San Miguel, A new method for the analysis of plasma cell DNA content in multiple myeloma samples using a CD38/propidium iodide double staining technique, Cytometry 17 (1994), 332-339.

[25] P.S. Rabinovitch, DNA content histogram and cell-cycle analysis, in: Methods in Cell Biology, Z. Darnzynkiewicz, J.P. Robinson and H.A. Crissman, eds, Vol. 41, Academic Press, San Diego, 1994, pp. 263-296.

[26] J.V. Watson, S.H. Chambers and P.J. Smith, A pragmatic approach to the analysis of DNA histograms with a definable G1 peak, Cytometry 8 (1987), 1-8.

[27] M.G. Ormerod, A.W.R. Payne and J.V. Watson, Improved program for the analysis of DNA histograms, Cytometry 8 (1987), 637-641. 


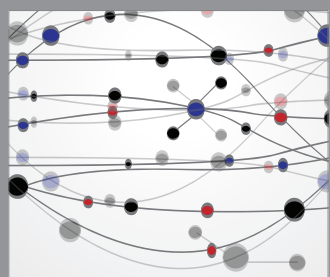

The Scientific World Journal
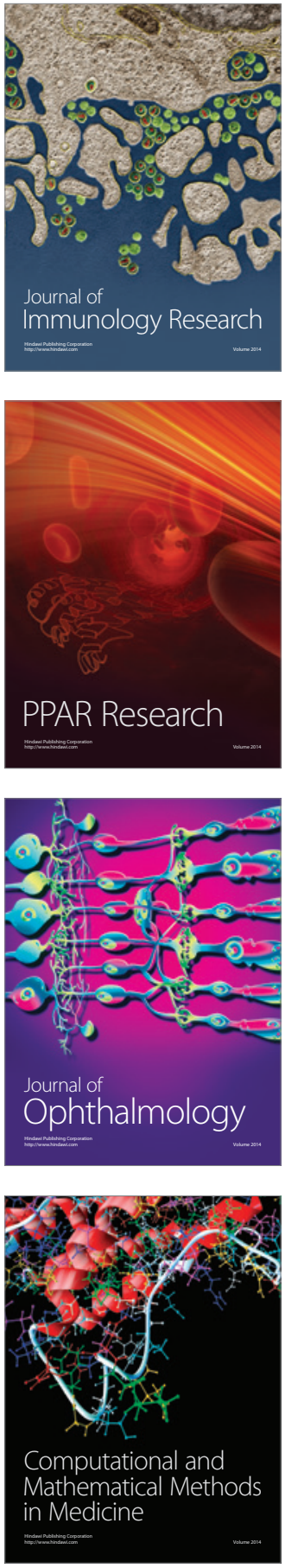



Gastroenterology

Research and Practice
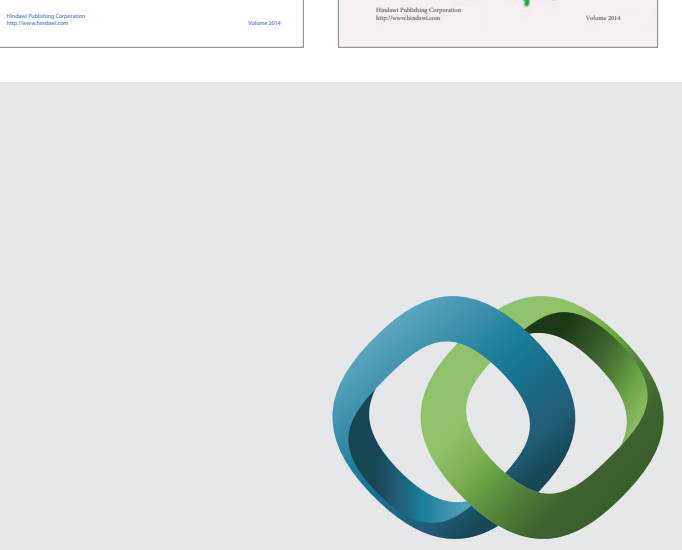

\section{Hindawi}

Submit your manuscripts at

http://www.hindawi.com
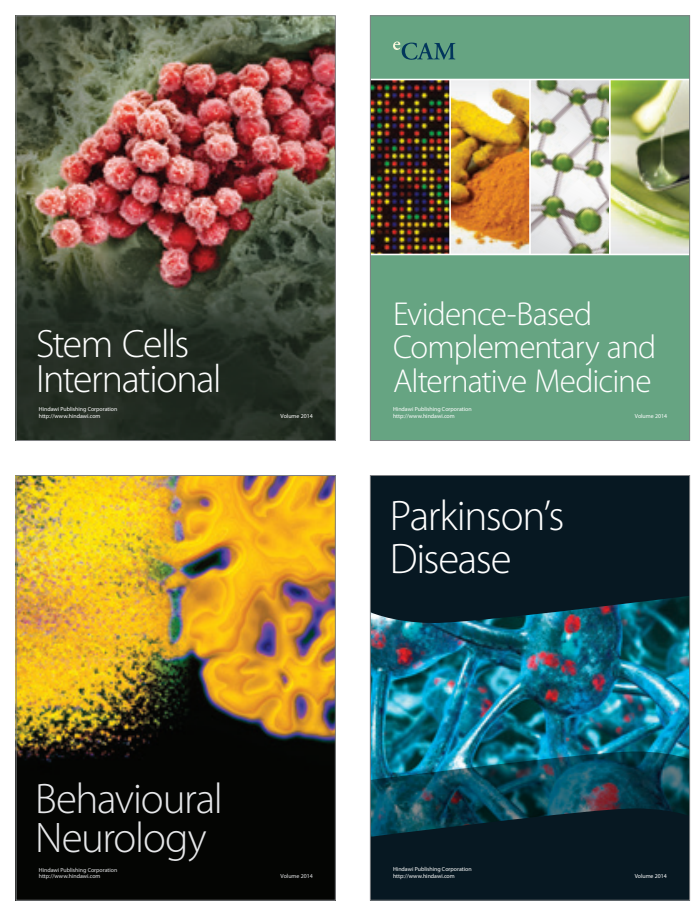

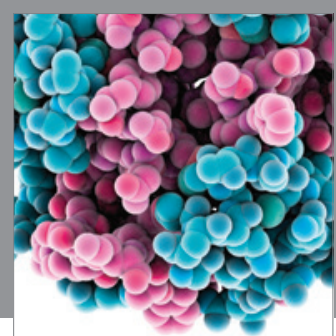

Journal of
Diabetes Research



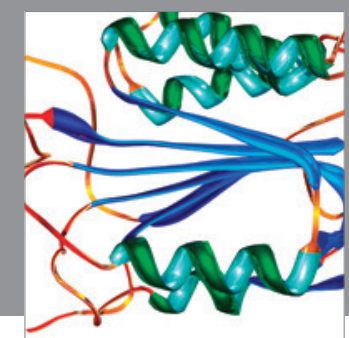

Disease Markers
